\title{
A metabolic grid among versiconal hemiacetal acetate, versiconol acetate, versiconol and versiconal during aflatoxin biosynthesis
}

\author{
KIMIKo YABE, ${ }^{1 *}$ YoShIJI ANDO $^{1}$ and TAKaSHI HAMASAKI ${ }^{2}$ \\ ${ }^{1}$ National Institute of Animal Health, Tsukuba, Ibaraki 305, Japan \\ ${ }^{2}$ Faculty of Agriculture, Tottori University, Tottori 680, Japan
}

(Received 12 February 1991; revised 3 June 1991; accepted 18 June 1991)

\begin{abstract}
Dichlorvos treatment of aflatoxigenic Aspergillus parasiticus SYS-4 (NRRL 2999) or a versicolorin Aaccumulating mutant, NIAH-9, resulted in accumulation of versiconol acetate (VOAc) and versiconal hemiacetal acetate (VHA), whereas the production of aflatoxins, versicolorin A (VA), and versiconol (VOH) decreased. In feeding experiments using another non-aflatoxigenic mutant, NIAH-26, aflatoxins were newly produced from each of VHA, VOAc, VOH, versicolorin B (VB) and versicolorin C (VC). In these experiments, aflatoxin production from VHA or VOAc was inhibited by dichlorvos, whereas that from each of VOH, VB and VC was insensitive to dichlorvos. In cell-free experiments using the cytosol fraction of NIAH-26, VHA was converted to VC (or VB) and a substance tentatively identified as versiconal (VHOH). By further addition of NADH or NADPH to the same reaction mixture, VOAc and VOH were also formed together with VC (VB) and VHOH. VOH was produced from VOAc irrespective of nicotinamide adenine nucleotide. Also, the incubation of VOH in the presence of NAD or NADP led to the formation of VC (VB). The production of VC (VB) and VHOH from VHA, and that of VOH from VOAc was inhibited by dichlorvos, whereas the production of VOAc from VHA, and that of VC (VB) from VOH, was insensitive to dichlorvos. These results indicate that a metabolic grid catalysed by dehydrogenase and esterase among VHA, VOAc, VOH and VHOH, and a reaction from VHOH to VC (VB) are involved in aflatoxin biosynthesis. These enzyme activities were also detected when yeast extract peptone medium was used, or when $A$. oryzae SYS-2 was examined.
\end{abstract}

\section{Introduction}

Aflatoxins are toxic secondary metabolites produced by certain strains of the common moulds Aspergillus flavus and $A$. parasiticus. The biosynthetic pathway of aflatoxins is generally accepted to be as follows: acetate/malonate $\rightarrow$ norsolorinic acid (NA) $\rightarrow$ averantin $\rightarrow$ averufin $\rightarrow$ versiconal hemiacetal acetate $(\mathrm{VHA}) \rightarrow$ versicolorin $\mathrm{A}$ (VA) $\rightarrow$ sterigmatocystin (ST) $\rightarrow O$-methylsterigmatocystin $(\mathrm{OMST}) \rightarrow$ aflatoxin $\mathrm{B}_{1}\left(\mathrm{AFB}_{1}\right)$ (Bennett \& Christensen, 1983; Bhatnagar et al., 1987; Dutton, 1988; Yabe et $a l ., 1988 b$ ). Fig. 1 shows the chemical structure of some of these compounds. VHA accumulates in aflatoxigenic strains in the presence of the organophosphorus insecti-

Abbreviations: $\mathrm{AFB}_{1}, \mathrm{AFB}_{2}, \mathrm{AFG}_{1}, \mathrm{AFG}_{2}$, aflatoxins $\mathrm{B}_{1}, \mathrm{~B}_{2}, \mathrm{G}_{1}$, $\mathrm{G}_{2}$ : NA, norsolorinic acid; NEM, $N$-ethylmaleimide; $\mathrm{ST}$, sterigmatocystin; VA, VB, VC, versicolorins A, B, C; VAAc, versicolorin A hemiacetal acetate; VAOH, versicolorin A hemiacetal; VHA, versiconal hemiacetal acetate; $\mathrm{VHOH}$, versiconal; VOAc, versiconol acetate; $\mathrm{VOH}$, versiconol; YEP, yeast extract peptone; YES, yeast extract sucrose. cide dichlorvos (dimethyl 2,2-dichlorovinylphosphate) (Schroeder et al., 1974; Yao \& Hsieh, 1974). VHA is known to be a key substance for understanding how the linear C6 side-chain of NA is converted into the branched $\mathrm{C} 4$ unit on the bisfuran ring system of VA, ST and aflatoxins.

It has been reported that in addition to VHA, dichlorvos treatment of aflatoxigenic strains results in the accumulation of versiconol acetate (VOAc) and versiconol (VOH) (Steyn et al., 1979), as well as versicolorin C (VC), versicol, averufin, averufanin, VOH and versicol acetate (Dutton \& Anderson, 1980). Recently, Hsieh et al. (1989) and Anderson \& Chung (1990) have independently reported that VHA was converted to VC (or VB) through the formation of versiconal (VHOH) in cell-free systems; since $\mathrm{VC}$ is a racemate of $V B$, and these substances were not separated by TLC, they could not identify whether the product was VB or VC. VB, VC and VOH are natural metabolites of Aspergillus versicolor (Hamasaki et al., 1965, 1967; Hatsuda et al., 1969), whilst VOAc and VOH are also 


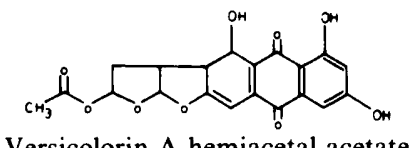

Versicolorin A hemiacetal acetate (VAAc)<smiles>C=C(C)OCCOC1Oc2cc3c1c(O)c2c(=O)c1c(C)cc(O)cc1c3=O</smiles>

Versiconal hemiacetal acetate (VHA)

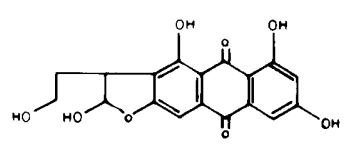

Versiconal (VHOH)

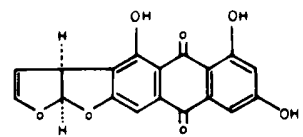

Versicolorin A (VA)

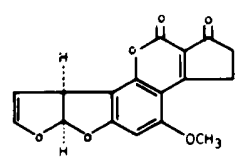

Aflatoxin $B_{1}$

$\left(\mathrm{AFB}_{1}\right)$

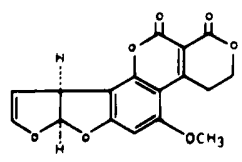

Aflatoxin $G_{1}$

$\left(\mathrm{AFG}_{1}\right)$

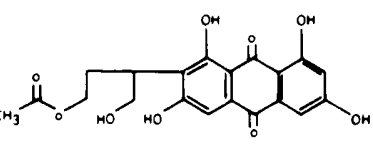

Versiconol acetate (VOAc)

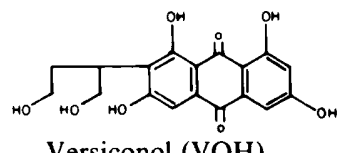

Versiconol (VOH)

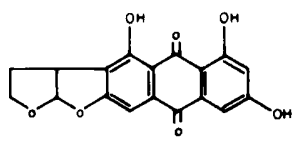

Versicolorin C (VC)

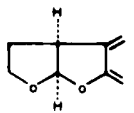

Versicolorin B

(VB)

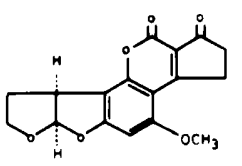

Aflatoxin $\mathrm{B}_{2}$

$\left(\mathrm{AFB}_{2}\right)$

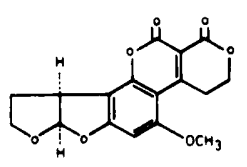

Aflatoxin $\mathrm{G}_{2}$

$\left(\mathrm{AFG}_{2}\right)$

Fig. 1. Structures of aflatoxins and probable precursors.

natural metabolites of Bipolaris sorokiniana (Maes \& Steyn, 1984). Little information is available, however, on the interrelationships among these compounds and their involvement in aflatoxin biosynthesis. In this study, we examined the biosynthetic relationship of VOAc and VOH to VHA, VHOH and VC (or VB) using both cellfree and culture systems.

\section{Methods}

Micro-organisms. Aflatoxin-producing wild strain $A$. parasiticus SYS-4 (NRRL 2999) and two kinds of UV-irradiated mutants of $A$. parasiticus SYS-4, NIAH-26 and NIAH-9, were used (Yabe $e t$ al., 1988a). NIAH-26 produces neither aflatoxins nor precursors, and NIAH-9 is a VA-accumulating mutant which does not produce aflatoxins. Aflatoxin-non-producing wild strain A. oryzae SYS-2 (IFO 4251) was also used.

Media and growth conditions. For the preparation of conidiospores, either $\boldsymbol{A}$. parasiticus or $\boldsymbol{A}$. oryzae was grown on malt extract agar medium at $28^{\circ} \mathrm{C}$ for 1 week, and conidiospores were then collected by filtration using $30 \mu \mathrm{M}$ nylon mesh (Yabe et al., 1987). Malt extract was prepared by boiling $50 \mathrm{~g}$ ground malt in 1 litre distilled water for $30 \mathrm{~min}$, and then filtering the extract through Miracloth (CalbiochemBehring); the filtered extract was supplemented with $30 \mathrm{~g}$ glucose, $3 \mathrm{~g}$ peptone and $3 \mathrm{~g}$ agar.

YES liquid medium $[2 \%(w / v)$ yeast extract, $20 \%(w / v)$ sucrose $]$ was used as aflatoxin-inducing medium, whilst YEP liquid medium $[2 \%$ $(w / v)$ yeast extract, $20 \%(w / v)$ peptone] was used as aflatoxin-noninducing medium (Abdollahi \& Buchanan, 1981).

Standard samples of metabolites. VHA and VOAc were extracted with acetone from mycelia of the mutant strain NIAH-9, which had been cultured in YES medium supplemented with 100 p.p.m. dichlorvos $\left(0.14 \mathrm{mg} \mathrm{ml}^{-1}\right)$. The extract was then purified by Sephadex LH 20 column chromatography and preparative silica gel TLC. VA, VB, VC and VOH were prepared from mycelia of $A$. versicolor (Vuill.) Tiraboschi (Hamasaki et al., 1965, 1967; Hatsuda et al., 1969). Versicolorin A hemiacetal acetate (VAAc) was chemically synthesized by treatment of VA with acetic acid, and then purified by Sephadex $\mathrm{LH}$ 20 chromatography and preparative silica gel TLC. Commercially prepared standards of $\mathrm{AFB}_{1}, \mathrm{AFB}_{2}, \mathrm{AFG}_{1}$ and $\mathrm{AFG}_{2}$ (Makor Chemicals) were used for comparison. The concentration of the metabolites in methanol was determined from UV absorption spectra using molar absorption coefficients (Hamasaki, 1965; Hatsuda et al., 1969; Steyn et al., 1979; Anderson \& Dutton, 1980; Cole \& Cox, 1981) as follows $\left(\mathrm{M}^{-1} \mathrm{~cm}^{-1}\right)$ : VHA $(480 \mathrm{~nm}), 7250$; VOAc $(453 \mathrm{~nm}), 8500$; VOH $(455 \mathrm{~nm}), 7400$; VA $(452 \mathrm{~nm}), 8166$; VB $(450 \mathrm{~nm}), 8700$; VC $(450 \mathrm{~nm}), 10700$; VAAc $(459 \mathrm{~nm}), 7100 ;$ AFB $_{1}(362 \mathrm{~nm}), 21800$; $\mathrm{AFB}_{2}(363 \mathrm{~nm}), 24000 ; \mathrm{AFG}_{1}(362 \mathrm{~nm}), 16100 ; \mathrm{AFG}_{2}(365 \mathrm{~nm})$, 19300.

Inhibition and feeding experiments. The tip culture method was used for producing and characterizing the aflatoxins and their precursors. A $1 \mathrm{ml}$ Pipetman tip (Gilson) was employed as the culture vessel (Yabe $e t$ al., 1988a). A. parasiticus SYS-4 or the mutant strain NIAH-9 was incubated for $4 \mathrm{~d}$ in YES or YEP medium supplemented with $0,3,10$, 30,100 or 300 p.p.m. dichlorvos. Since precursor pigments of aflatoxins accumulated in the mycelia, the mycelia were extracted with acetone and the extract was dried. The resulting residue was solubilized with $0.2 \mathrm{ml}$ benzene/acetonitrile $(98: 2, \mathrm{v} / \mathrm{v})$. A $50 \mu \mathrm{l}$ sample of this solution was then spotted on to a silica gel TLC plate. The pigments were analysed by TLC with a developing solution composed of chloroform/ ethyl acetate $/ 90 \%(w / w)$ formic acid $(6: 3: 1$, by vol.), and conventional photographs of the TLC plate were taken.

Feeding experiments (Yabe et al., 1988b) with VHA, VOAc, VOH, VB or VC $(20 \mu \mathrm{M}$ each), were carried out in the absence or presence of 100 p.p.m. dichlorvos. Since aflatoxins were excreted from mycelia into the medium, $25 \mu \mathrm{l}$ culture medium was directly spotted on to a silica gel TLC plate after $4 \mathrm{~d}$ culture, and then examined by TLC using the developing solution described above. The production of some aflatoxin precursors from the substances added was also examined by analysing the acetone extract of the mycelia. Fluorescence photographs of the TLC plate were taken.

Cell-free studies. Post-mitochondrial fractions were prepared from the mycelia of $A$. parasiticus NIAH-26 and $A$. oryzae SYS-2 (Yabe $e t$ al., $1988 \mathrm{~b}$ ). The cytosol (post-microsome) fraction of NIAH-26 was further purified by centrifuging the post-mitochondrial fraction at $105000 \mathrm{~g}$ for $90 \mathrm{~min}$. To the resultant post-mitochondrial and cytosol fractions, glycerol was added (final concentration $10 \%, \mathrm{v} / \mathrm{v}$ ), and the fractions were stored at $-80^{\circ} \mathrm{C}$ until use. The protein concentration was determined by the method of Bradford (1976).

To examine the effect of $N$-ethylmaleimide (NEM) on enzyme 
activities, the cytosol fraction of NIAH-26 $\left(2 \cdot 3 \mathrm{mg}\right.$ protein $\left.\mathrm{ml}^{-1}\right)$, which had been cultured in YES medium, was incubated with $11 \mathrm{~mm}$ NEM at $37^{\circ} \mathrm{C}$ for $30 \mathrm{~min}$ in a mixture containing $50 \mathrm{~mm}$-potassium phosphate buffer $(\mathrm{pH} 7.5)$ and $12.5 \%(\mathrm{v} / \mathrm{v})$ glycerol. The reaction was started by the addition of NEM and stopped by the addition of $2 \mathrm{M}-2-$ mercaptoethanol (final concentration $10 \%, \mathrm{v} / \mathrm{v}$ ).

Samples of cytosol $\left(0.8 \mathrm{mg}\right.$ protein $\left.\mathrm{ml}^{-1}\right)$, NEM-treated cytosol $\left(0.8 \mathrm{mg}\right.$ protein $\left.\mathrm{ml}^{-1}\right)$ and post-mitochondrial fractions were each incubated in a reaction mixture containing $60 \mathrm{~mm}$-potassium phosphate buffer $(\mathrm{pH} 7.5), 10 \%(\mathrm{v} / \mathrm{v})$ glycerol, and $60 \mu \mathrm{M}$ each of VHA, VOAc, VOH and VAAc. When specified, dichlorvos (200 p.p.m.) or $2 \mathrm{~mm}$ each of NADH, NAD, NADPH and NADP was added to the reaction mixture (total volume $50 \mu \mathrm{l}$ ). After incubation at $37^{\circ} \mathrm{C}$ for $1 \mathrm{~h}$, the reaction was terminated by the addition of $3 \mu \mathrm{l}$ formic acid $(99 \%$, $\mathrm{w} / \mathrm{w})$ and mixing with a Vortex mixer. After centrifugation at $10000 \mathrm{~g}$ for $2 \mathrm{~min}, 25 \mu \mathrm{l}$ of the supernatant was spotted on to a silica gel plate, and the reaction products were analysed by TLC as described above. Fluorescence photographs of the products were taken.

Photographic technique. Conventional photographs of the TLC plates for various anthraquinone pigments were taken using a Contax 167 camera with a Schott BG 12 filter on Kodak technical pan film 2415 developed in Kodak D-11 high-contrast developer. Fluorescence photographs of aflatoxins and their precursors in feeding experiments were taken using a Funa UV light (type SL-800F, $365 \mathrm{~nm}$ ) with Shott K V 450 and BG 12 filters. Fluorescence photographs of cell-free studies were taken using a Funa UV light (type SL-800F, $365 \mathrm{~nm}$ ) and a Contax 167 camera with Kenko SL-39 UV and SO 56.2 (YA-3) filters on Kodak $T_{\max } 400 \mathrm{film}$. Two filters were used to detect red, orange, and yellow fluorescent products ciearly.

\section{Results}

\section{Accumulation of VHA and VOAc in cultures of $A$. parasiticus treated with dichlorvos}

The VA-accumulating mutant, NIAH-9, was grown in YES medium for $4 \mathrm{~d}$ in the presence of $0,3,10,30,100$, and 300 p.p.m. dichlorvos, and then mycelial extracts were subjected to TLC against authentic standards of VA, VHA, VOAc and VOH (Fig. 2). In the presence of dichlorvos, metabolites with the same chromatographic mobilities as VA and VOH decreased while metabolites with the same mobilities as VHA and VOAc accumulated. This accumulation was noted after $3 \mathrm{~d}$ with 100 p.p.m. dichlorvos (Fig. 2b, lane 2). The same results were obtained when wild-type $A$. parasiticus SYS-4 was incubated with dichlorvos with the exception that aflatoxins, instead of VA, decreased. None of these metabolites were observed in the control group of the wild-type and VA-accumulating mutant in YEP, nor in wild-type $A$. oryzae grown in YES in the presence of dichlorvos.

The mutant $A$. parasiticus NIAH-26 was cultured in YES medium for $4 \mathrm{~d}$ in the presence of VHA, VOAc, $\mathrm{VOH}, \mathrm{VB}$ or $\mathrm{VC}$, and then the culture medium was subjected to TLC against authentic standards of aflatoxins (Fig. 3). Aflatoxins were produced from all sub-

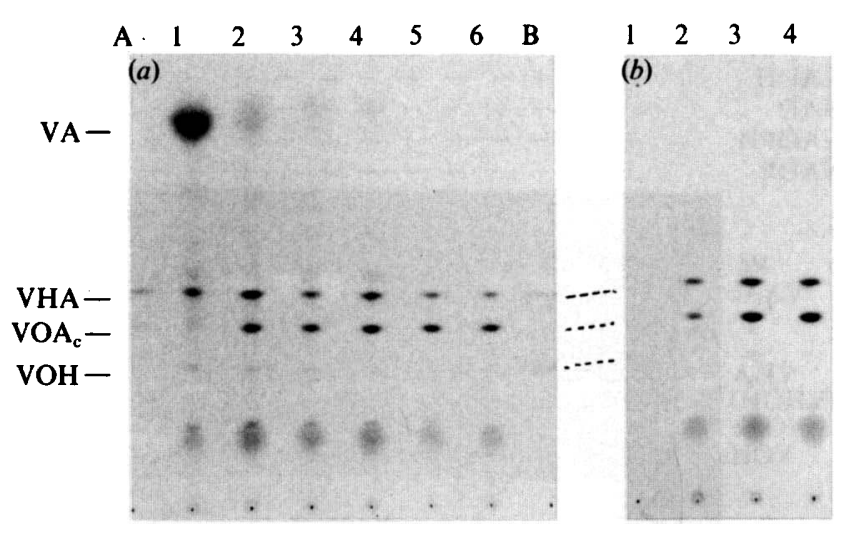

Fig. 2. Conventional photographs of thin-layer chromatograms of mycelial extracts of dichlorvos-treated cultures of a VA-accumulating mutant, A. parasiticus NIAH-9. Cultures were treated with (a) 0 (lane 1), 3 (lane 2), 10 (lane 3), 30 (lane 4), 100 (lane 5) and 300 (lane 6) p.p.m. dichlorvos, and with (b) 100 p.p.m. dichlorvos for 2 (lane 1), 3 (lane 2), 4 (lane 3) and 5 (lane 4) d. Lane A, authentic standards of VHA and VOAc; lane B, authentic standards of VHA and VOH.

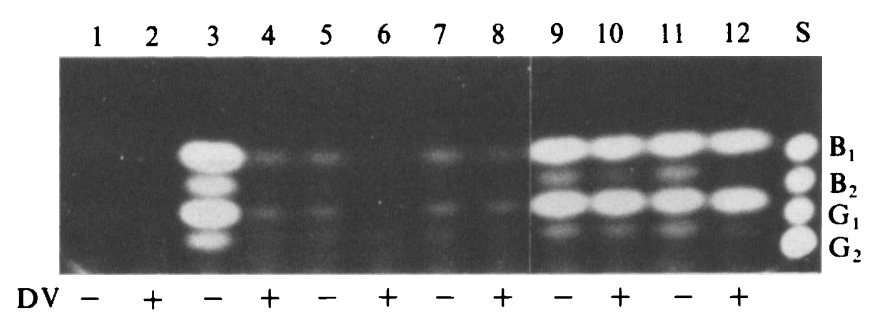

Fig. 3. Fluorescence photographs showing production of aflatoxins from various substances added to YES medium in feeding experiments using $A$. parasiticus NIAH-26. Dichlorvos (DV, 100 p.p.m.) was added as indicated $(+)$. Lanes: 1 and 2 , no supplement; 3 and 4 , VHA; 5 and 6 , VOAc; 7 and 8 , VOH; 9 and $10, \mathrm{VB} ; 11$ and $12, \mathrm{VC} ; \mathrm{S}$, authentic standards of $A_{1} B_{1}\left(B_{1}\right), A F B_{2}\left(B_{2}\right), A_{1}\left(G_{1}\right), A F G_{2}\left(G_{2}\right)$.

different. In the presence of dichlorvos, the production of aflatoxins from VHA and VOAc was inhibited, whereas that from VB, VC and VOH was insensitive to dichlorvos. When mycelial extracts were examined by TLC, the accumulation of $\mathrm{VOH}$ from exogenous VOAc, and of VC (VB) from exogenous VHA, was observed. These conversions [VOAc $\rightarrow \mathrm{VOH}$ and $\mathrm{VHA} \rightarrow \mathrm{VC}(\mathrm{VB})$ ] were inhibited by dichlorvos (data not shown). No aflatoxins were formed in the control of this mutant grown in YEP.

\section{Production of $V C(V B)$ from $V H A, V O A c$ and $V O H$ in the cell-free system}

The cytosol fraction of NIAH-26 mycelia cultured in YES was incubated with VHA, VOAc or VOH. Two orange fluorescent substances were formed from VHA, and the $R_{F}$ value of the upper spot corresponded to VC 
$\begin{array}{lllllllllllllllll}\text { A } & \text { B } & 1 & 2 & 3 & 4 & 5 & 6 & 7 & 8 & 9 & 10 & 11 & 12 & 13 & \text { A } & \text { B }\end{array}$
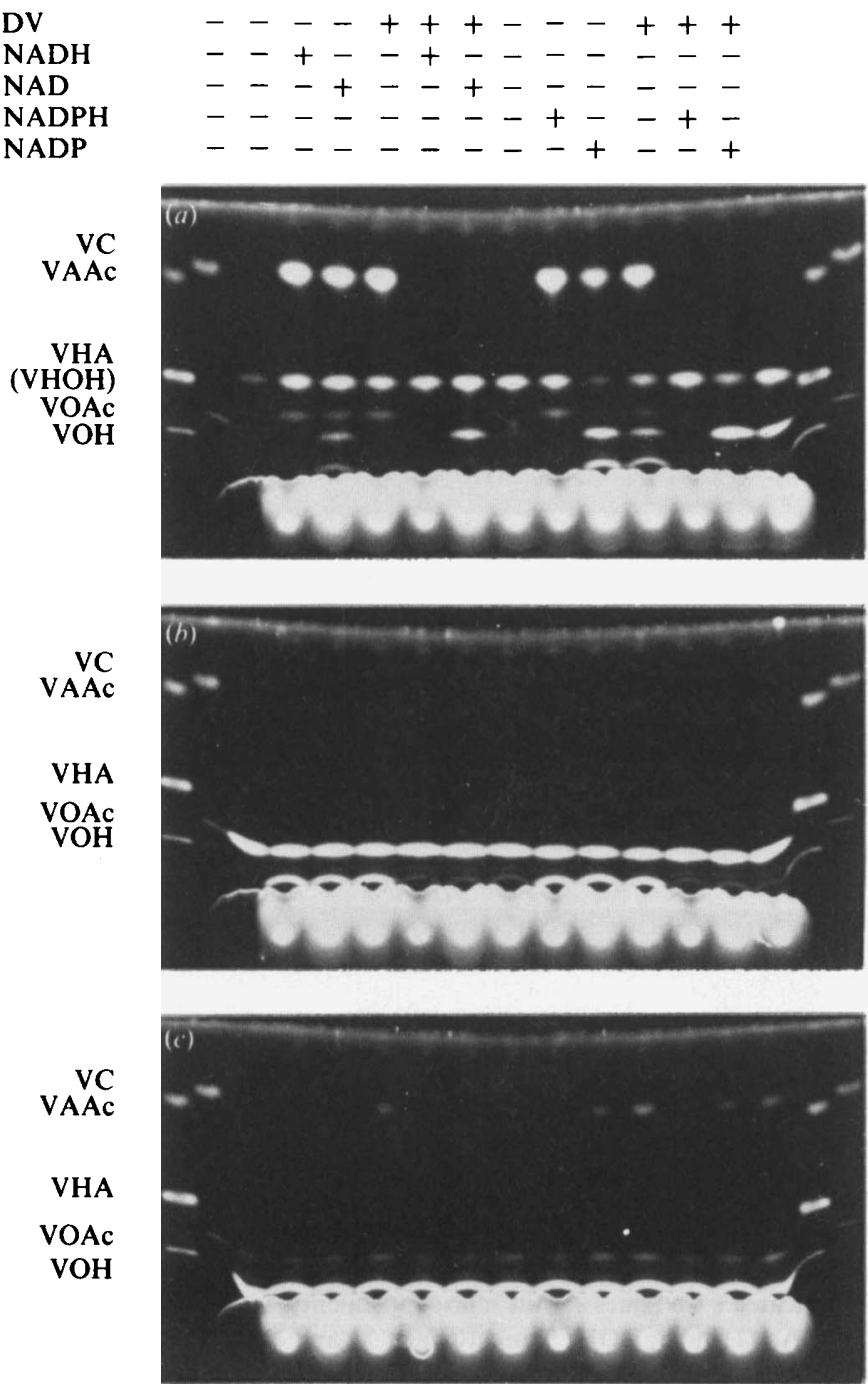

(d)

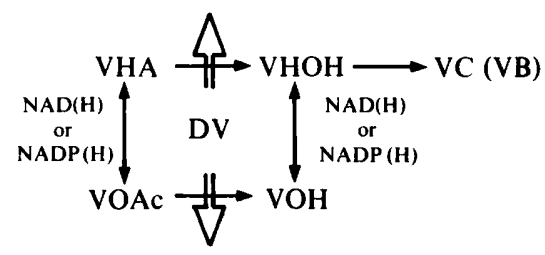

Fig. 4. Fluorescence photographs showing enzymic conversions among VHA, VOAc, VOH, VHOH and VC by the cytosol fraction of $A$. parasiticus NIAH-26. Substrate: (a) VHA, (b) VOAc, (c) VOH. Lanes: 1, no cytosol; 2-13, inhibitor and cofactors, dichlorvos (DV, 200 p.p.m.), and NADH, NAD, NADPH and NADP (2 mM each) were added as indicated (+). Lane A: authentic standards of VAAc, VHA and $\mathrm{VOH}$; lane $\mathrm{B}$, authentic standards of VC and VOAc. (d) Proposed metabolic grid interconnecting anthraquinone pigments.

(or VB) (Fig. 4a, lanes 2 and 8). Though VB and VC could not be separated by TLC analysis, the product is referred to as VC for simplicity. When the lower spot, immediately under VHA, was extracted with ethyl

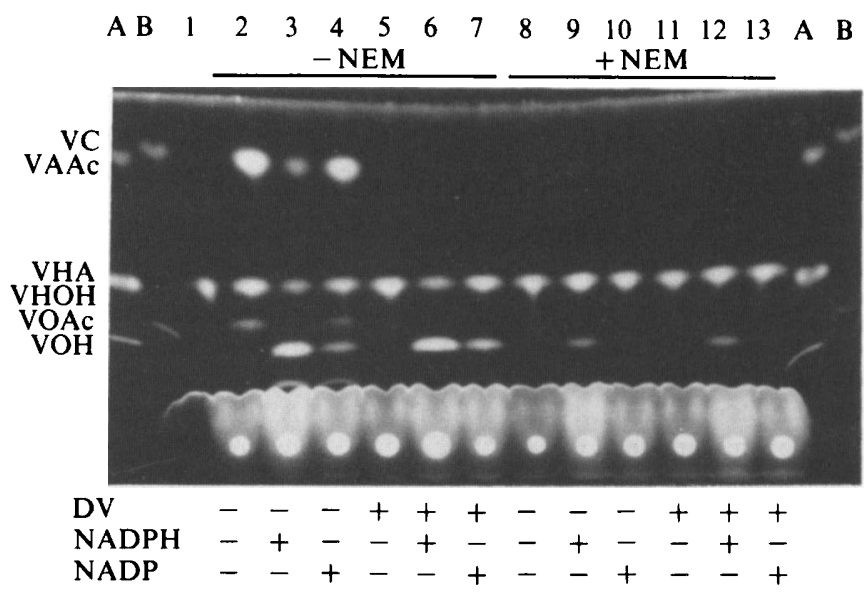

Fig. 5. Fluorescence photographs showing the effect of NEM treatment of the cytosol fraction of $A$. parasiticus NIAH-26 on enzymic conversions from VHA to VOAc, VOH, VHOH and VC. Lanes: 1, no cytosol; 2-7, NEM-non-treated cytosol; 8-13, NEM-treated cytosol. Inhibitor and cofactors, dichlorvos (DV, 200 p.p.m.), and NADPH and NADP ( $2 \mathrm{mM}$ each) were added as indicated $(+)$. Lane A: authentic standards of VAAc, VHA and VOH; lane B, authentic standards of VC and VOAc.

acetate and re-analysed by TLC, using the same developing solution, this spot disappeared, whilst $\mathrm{VC}$ was newly formed (data not shown). Since this substance, corresponding to the lower spot, was not chloroform-extractable (Hsieh et al., 1989), it was considered to be versiconal ( $\mathrm{VHOH}$ ).

Addition of NADH or NADPH to the same reaction mixture led to the additional formation of two other substances, with the same chromatographic mobilities as VOAc and VOH, respectively (Fig. $4 a$, lanes 3 and 9). Though VOAc and $\mathrm{VOH}$ were also formed in the presence of NADP (Fig. 4a, lane 10), unidentified cytosol dehydrogenase which catalysed the reversible reactions between NADP and NADPH, or NAD and NADH (data not shown), probably contributed to these NADP-dependent reactions. The production of VC, VHOH and VOH from VHA was inhibited by the addition of dichlorvos to the reaction mixtures (Fig. $4 a$, lanes 5-7 and 11-13), whereas the production of VOAc from VHA was insensitive to dichlorvos (Fig. $4 a$, lanes 6 , 12 and 13 ).

When VOAc was incubated with the cytosol fraction, VOH was produced (Fig. $4 b$ ). This reaction was inhibited by dichlorvos irrespective of the presence of $\mathrm{NADP}(\mathrm{H})$ or $\mathrm{NAD}(\mathrm{H})$. When the same fraction was incubated with $\mathrm{VOH}$ in the presence of NAD or NADP, a small amount of $\mathrm{VC}$ formed irrespective of the presence of dichlorvos (Fig. 4c). On the other hand, no new product was found when VC was incubated with the cytosol fraction of $A$. parasiticus NIAH-26.

None of the reactions described here occurred when the cytosol fraction was heat-treated. 


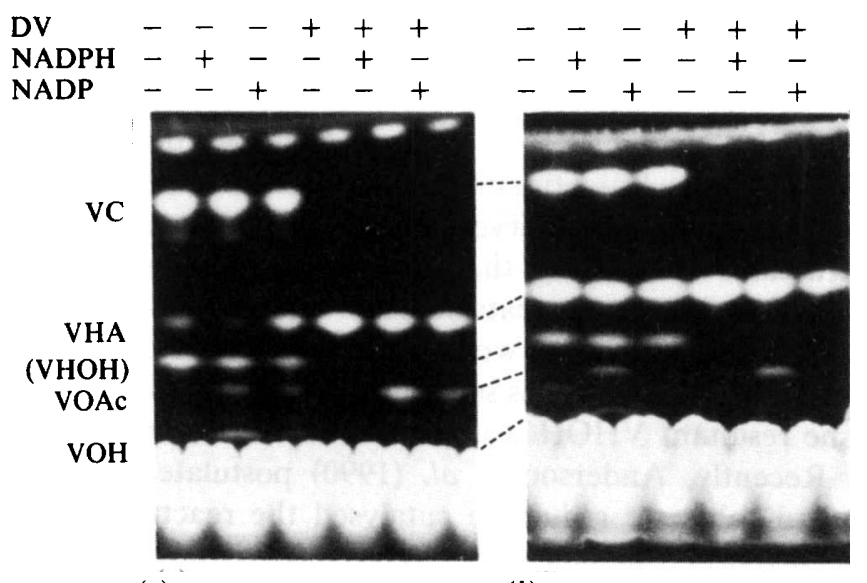

(a)

(b)

Fig. 6. Fluorescence photographs showing the enzymic conversion from VHA to other substances by the cell extract of $A$. parasiticus NIAH-26 which had been cultured in YEP medium $(a)$ or the extract of A. oryzae SYS- 2 which had been cultured in YES medium (b). Protein concentrations: (a) $3 \cdot 1 \mathrm{mg} \mathrm{ml}^{-1}$; (b) $2 \mathrm{mg} \mathrm{ml}^{-1}$. Inhibitor and cofactors, dichlorvos (DV, 200 p.p.m.), and NADPH and NADP ( $2 \mathrm{mM}$ each) were added as indicated $(+)$.

When the cytosol fraction of NIAH-26 was modified by the addition of NEM, a reagent for the modification of protein $\mathrm{SH}$ groups, the production of $\mathrm{VC}$ and $\mathrm{VHOH}$ from VHA, and that of VOAc from VHA, decreased (Fig. 5), and the production of $\mathrm{VOH}$ from VOAc also decreased (data not shown).

\section{Production of $V C(V B)$ in cell-free systems prepared either from moulds cultured in YEP medium or from the non-aflatoxigenic strain}

When either the post-mitochondrial fraction (Fig. $6 a$ ) or the cytosol fraction of NIAH-26, cultured in YEP medium, was incubated with VHA, similar results to those shown in Fig. 4 were obtained; $\mathrm{VHOH}$ and VC (VB) were produced from VHA, and VOAc and VOH were also formed in the presence of NADPH or NADP. The production of $\mathrm{VC}, \mathrm{VHOH}$ and $\mathrm{VOH}$ was inhibited by dichlorvos, whereas the production of VOAc from VHA was insensitive to dichlorvos. Similar results were also observed when either the post-mitochondrial fraction (Fig. $6 b$ ) or the cytosol of A. oryzae SYS-2 (IFO 4251), cultured in YES medium, was used.

\section{Formation of $V C(V B)$ from $V A A c$ by the cytosol fraction}

When the cytosol fraction of NIAH-26 cultured in YES medium was incubated with VAAc, VC (or VB) was formed depending on the presence of NADH or NADPH (Fig. 7). These reactions were inhibited by dichlorvos.
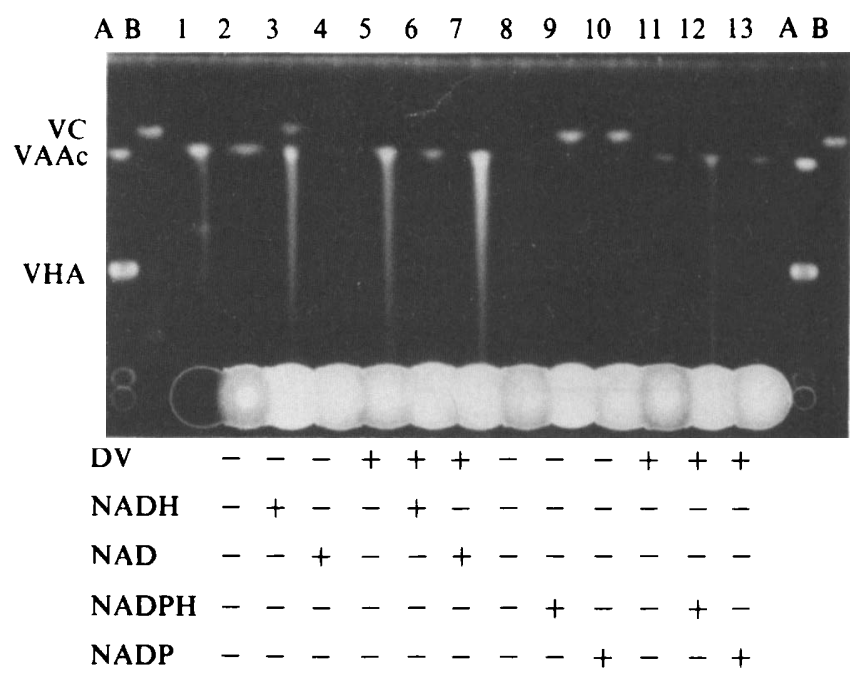

Fig. 7. Fluorescence photograph showing the production of VC from VAAc by the cytosol fraction of $A$. parasiticus NIAH-26. Lanes: 1, no cytosol ; 2-13, inhibitor and cofactors, dichlorvos (DV, 200 p.p.m.), and NADH, NAD, NADPH and NADP ( $2 \mathrm{mM}$ each) were added as indicated $(+)$. Lane $A$, authentic standards of VAAc and VHA; lane $B$, authentic standard of VC.

\section{Discussion}

The relationship of $\mathrm{VOAc}$ and $\mathrm{VOH}$ to aflatoxin biosynthesis had not previously been clarified, although a case for their intermediacy in aflatoxin biosynthesis had been strongly supported (Hatsuda et al., 1969; Maes $\&$ Steyn, 1984). On the other hand, the reaction VHA $\rightarrow$ $\mathrm{VHOH} \rightarrow \mathrm{VC}(\mathrm{VB})$ had also been reported (Hsieh et al., 1989; Anderson \& Chung, 1990). We showed in this study that VOAc and VOH as well as VB, VC and VHA were precursors of aflatoxins, that a metabolic grid operates among VHA, VOAc, VOH and VHOH during aflatoxin biosynthesis, and that the resultant $\mathrm{VHOH}$ is converted to VC (VB). We have also shown previously that $A F B_{1} \cdot A_{F G}$ and $A F B_{2} \cdot A_{F G}$ were independently produced from demethylsterigmatocystin (DMST) and dihydrodemethylsterigmatocystin (DHDMST), respectively (Yabe et al., 1989), that VB, which is a half component of VC, was converted to VA by a desaturase reaction, and that $\mathrm{VA}$ and $\mathrm{VB}$ served as precursors of $\mathrm{AFB}_{1} \cdot \mathrm{AFG}_{1}$ and $\mathrm{AFB}_{2} \cdot \mathrm{AFG}_{2}$, respectively (Yabe et al., $1991 \mathrm{~b}$ ). The proposed metabolic scheme for the late stages of aflatoxin biosynthesis is summarized in Fig. 8.

VHA and VOAc accumulate in mycelia following treatment of aflatoxigenic or VA-producing moulds with dichlorvos (Fig. 2). We observed that the production of aflatoxins from either VHA or VOAc, of VC from VHA, and of VOH from VOAc was inhibited by dichlorvos in the feeding experiments (Fig. 3). Also, the production of $\mathrm{VHOH}$ and VC from VHA, and of VOH from VOAc was inhibited by dichlorvos in the cell-free experiments. 

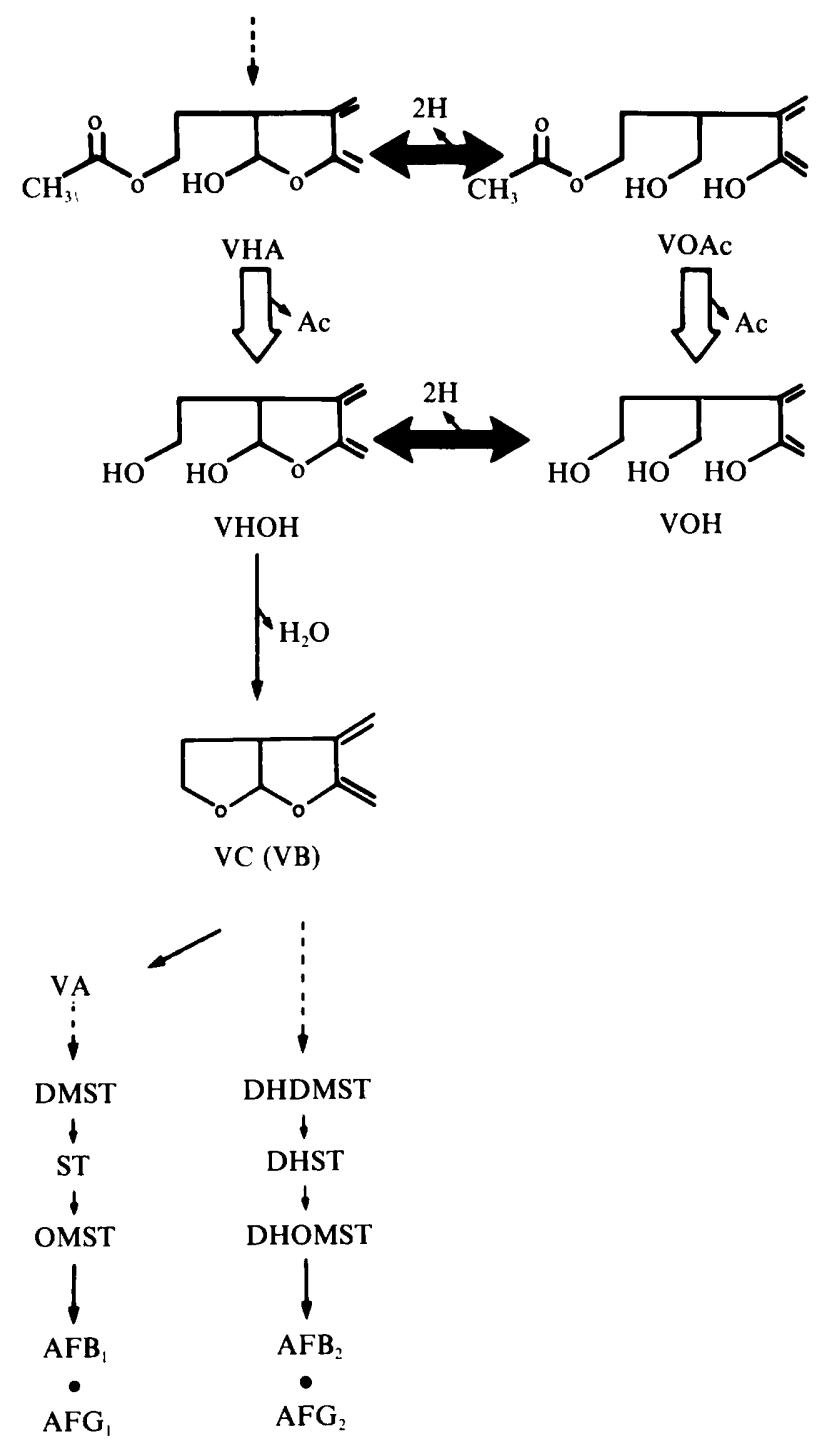

Fig. 8. Metabolic scheme proposed for the late stages of aflatoxin biosynthesis. Solid arrows, dehydrogenase reaction; open arrows, esterase reaction; solid thin arrows, confirmed reactions in this and previous papers (Bhatnagar et al., 1987; Hsieh et al., 1989; Anderson \& Chung, $1991 a$; Yabe et al., 1988a, 1989, $1991 a$, and this study; dashed arrows, unconfirmed reactions. Abbreviations: DHST, dihydrosterigmatocystin; DMST, demethylsterigmatocystin; DHDMST, dihydrodemethylsterigmatocystin; OMST, $O$-methylsterigmatocystin; DHOMST, dihydro- $O$-methylsterigmatocystin.

Thus, the step(s) that immediately follow(s) the formation of both VHA and VOAc, and those that precede the formation of $\mathrm{VC}$ and $\mathrm{VOH}$, may be blocked by dichlorvos. Based on the similarity of the structures of these substrates, the same post-microsomal enzyme, esterase, is likely to be involved in both reactions. This enzyme is sensitive to dichlorvos and NEM modification.
In the cell-free experiments, the production of VOAc from VHA was dependent on a reduced nicotinamide nucleotide, irrespective of the presence of dichlorvos, whereas the production of $\mathrm{VC}$ from $\mathrm{VOH}$ was dependent on an oxidized nicotinamide nucleotide, irrespective of the presence of dichlorvos. Based on the similarity of structures it is likely that the same cytosol enzyme, dehydrogenase, may catalyse the conversion between VOAc and VHOH as well as that between VOAc and VHA. This enzyme was sensitive to NEM modification. The resultant VHOH was converted to VC (or VB).

Recently, Anderson et al. (1990) postulated that a broad substrate reductase catalysed the reaction from versicolorin A hemiacetal (VAOH) to VC in the presence of NADPH. In the current study, we showed that versicolorin A hemiacetal acetate (VAAc) was converted to VC (or VB) in the presence of the reduced nicotinamide nucleotide by the cytosol, and that this reaction was inhibited by dichlorvos (Fig. 7). Also, the reaction from $\mathrm{VAOH}$ to $\mathrm{VC}$ (VB) was insensitive to dichlorvos in the preliminary experiments $(K$. Yabe, unpublished results). Therefore, the reductase suggested by Anderson et al. (1990) may catalyse the reactions from VAAc to VHA, and from VAOH to VHOH, and the resultant VHA and $\mathrm{VHOH}$ may be converted to $\mathrm{VC}$ through the metabolic grid and the reaction from $\mathrm{VHOH}$ to VC. However, since VAAc and VAOH were chemically synthesized from VA, and not isolated from moulds, the reduction of VAAc and VAOH might not be metabolically significant. On the other hand, the dehydrogenase activity reported in this paper catalysed a reversible reduction of the central hemiacetal structure in the furan ring of VHA into the hydroxy group of $\mathrm{VOH}$, and VHA and VOAc are natural metabolites of certain polyketide-synthesizing moulds.

The order of accumulation of VHA and VOAc could not be distinguished by treatment of the culture with dichlorvos (Fig. $2 b$ ). However, VHA was usually the major substance which accumulated during treatment with dichlorvos (Fig. 2), and the other substances in the metabolic grid were produced from VHA in the cell-free experiments (Fig. 4). The amounts of aflatoxins produced from VOAc and VOH were much smaller than that from VHA (Fig. 3). Hence, it is likely that VHA is the first substance at the entrance of the grid and that the pathway VHA $\rightarrow \mathrm{VHOH} \rightarrow \mathrm{VC}(\mathrm{VB})$ is the main pathway for aflatoxin biosynthesis.

Interestingly, both esterase and dehydrogenase were active in the cytosol fraction of NIAH-26 cultured in aflatoxin-non-inducible YEP medium. Also, both enzyme activities were detected in the cytosol fraction of the non-aflatoxigenic strain $A$. oryzae SYS-2. However, when $A$. parasiticus SYS-4 was cultured with dichlorvos in YEP medium or when $A$. oryzae SYS-2 was cultured 
with dichlorvos in YES medium, the accumulation of pigments such as VHA and VOAc was never observed. These results indicate that the metabolite(s) in the step(s) preceding the formation of VHA and VOAc is (are) not produced under these conditions. In fact, we determined that the activities of several enzymes involved in the former part of aflatoxin biosynthesis, that is the pathway from NA to averufin, were not detected under aflatoxinnon-inducible conditions (Yabe et al., 1991a). Consequently, the metabolic grid elucidated in this study operates only in aflatoxin-inducible conditions. The function of the esterase and the dehydrogenase under non-aflatoxigenic conditions remains unclear.

$\mathrm{VC}$ is a racemate and has been purified as a pair of molecules connected at the centre of symmetry (Fukuyama et al., 1975). VB, which is a diastereomer of VC, has the same stereo-specificity as aflatoxins. In the current study, we could not distinguish between VB and VC by TLC experiments, though our results revealed that both VB and VC were precursors of aflatoxins. A study of the chirality of the precursors during aflatoxin biosynthesis is now in progress in our laboratory.

We thank N. Terakado, National Institute of Animal Health, Ministry of Agriculture, Forestry and Fisheries, Japan, for critically reviewing this manuscript. This work was supported in part by a Grant-in-Aid (Bio Media Program) from the Ministry of Agriculture, Forestry and Fisheries, Japan (BMP91-III-2-3).

\section{References}

AbDollahi, A. \& Buchanan, R. L. (1981). Regulation of aflatoxin biosynthesis: induction of aflatoxin production by various carbohydrates. Journal of Food Science 46, 633-635.

Anderson, J. A. \& Chung, C. H. (1990). Conversion of versiconal acetate to versiconal and versicolorin $\mathrm{C}$ in extracts from Aspergillus parasiticus. Mycopathologia 110, 31-35.

Anderson, J. A., Chung, C. H. \& Cho, S.-H. (1990). Versicolorin A hemiacetal, hydroxydihydrosterigmatocystin, and aflatoxin $G_{2 a}$ reductase activity in extracts from Aspergillus parasiticus. Mycopathologia 111, 39-45.

ANDERSON, M. S. \& DUTTON, M. F. (1980). Biosynthesis of versicolorin A. Applied and Environmental Microbiology 40, 706-709.

Bhatnagar, D., McCormick, S. P., Lee, L. S. \& Hill, R. A. (1987). Identification of $O$-methylsterigmatocystin as an aflaroxin $\mathrm{B}_{1} / \mathrm{G}_{1}$ precursor in Aspergillus parasiticus. Applied and Environmental Microbiology 53, 1028-1033.

Bennett, J. W. \& Christensen, S. B. (1983). New perspectives on aflatoxin biosynthesis. Advances in Applied Microbiology 29, 53-92.

BRADFORD, M. M. (1976). A rapid and sensitive method for the quantitation of microgram quantities of protein utilizing the principle of protein-dye binding. Analytical Biochemistry 72, 248254.
Cole, R. J. \& Cox, R. H. (1981). Handbook of Toxic Fungal Metabolites, pp. 1-65 and 94-127. New York: Academic Press.

DutTON, M. F. \& ANDERSON, M. S. (1980). Inhibition of aflatoxin biosynthesis by organophosphorus compounds. Journal of Food Protection 43, 381-384.

Dutron, M. F. (1988). Enzymes and aflatoxin biosynthesis. Microbiological Reviews 52, 274-295.

FukuYama, K., Tsukihara, T., Katsube, Y., Hamasaki, T. \& Hatsuda, Y. (1975). The crystal and molecular structure of versicolorin $\mathrm{C}$, a metabolite of Aspergillus versicolor. Bulletin of the Chemical Society of Japan 48, 2648-2652.

Hamasaki, T., Hatsuda, Y., Terashima, N. \& Renbutsu, M. (1965). The structure of a new metabolite of Aspergillus versicolor. Agricultural and Biological Chemistry 29, 696-697.

Hamasaki, T., Hatsuda, Y., Terashima, N. \& Renbutsu, M. (1967). Studies on the metabolites of Aspergillus versicolor (Vuillemin) Tiraboschi. Part V. Isolation and structures of three new metabolites, versicolorins A, B and C. Agricultural and Biological Chemistry 31, 11-17.

Hatsuda, Y., Hamasaki, T., Ishida, M. \& Yashikawa, S. (1969). The structure of a new metabolite from Aspergillus versicolor. Agricultural and Biological Chemistry 33, 131-133.

Hsieh, D. P. H., WAN, C. C. \& Billington, J. A. (1989). A versiconal hemiacetal acetate converting enzyme in aflatoxin biosynthesis. Mycopathologia 107, 121-126.

Maes, C. M. \& Steyn, P. S. (1984). Polyketide-derived fungal metabolites from Bipolaris sorokiniana and their significance in the biosynthesis of sterigmatocystin and aflatoxin $\mathrm{B}_{1}$. Journal of the Chemical Society Perkin Transactions I 1984, 1137-1140.

SChroeder, H. W., Cole, R. J., GRisBY, R. D. \& HeIN, H., JR (1974). Inhibition of aflatoxin production and tentative identification of an aflatoxin intermediate "versiconal acetate" from treatment with dichlorvos. Applied Microbiology 27, 394-399.

Steyn, P. S., VlegaAr, R. \& Wessels, P. L. (1979). Structure and carbon-13 nuclear magnetic resonance assignments of versiconal acetate, versiconol acetate, and versiconol, metabolites from cultures of Aspergillus parasiticus treated with dichlorvos. Journal of the Chemical Society Perkin Transactions I 1979, 451-459.

Yabe, K., ANDo, Y., Ito, M. \& TeraKado, N. (1987). Simple method for screening aflatoxin-producing molds by UV photography. Applied and Environmental Microbiology 53, 230-234.

Yabe, K., Nakamura, H., ANDo, Y., Terakado, N., Nakajima H. \& HAMASAKI, T. (1988a). Isolation and characterization of Aspergillus parasiticus mutants with impaired aflatoxin production by a novel tip culture method. Applied and Environmental Microbiology 54, 20962100 .

YABE, K., ANDO, Y. \& HAMASAKI, T. (1988b). Biosynthesis relationship among aflatoxins $\mathrm{B}_{1}, \mathrm{~B}_{2}, \mathrm{G}_{1}$, and $\mathrm{G}_{2}$. Applied and Environmental Microbiology 54, 2101-2106.

Yabe, K., Ando, Y., Hashimoto, J. \& Hamasaki, T. (1989). Two distinct $O$-methyltransferases in aflatoxin biosynthesis. Applied and Environmental Microbiology 55, 2172-2177.

Yabe, K., Nakamura, Y., Nakajima, H., ANDo, Y. \& Hamasaki, T. $(1991 a)$. Enzymatic conversion of norsolorinic acid to averufin in aflatoxin biosynthesis. Applied and Environmental Microbiology 57, 1340-1345.

YABE, K., ANDO, Y. \& HAMASAKI, T. (1991 b). Desaturase activity in the branching step between aflatoxins $B_{1}$ and $G_{1}$ and aflatoxins $B_{2}$ and $\mathrm{G}_{2}$. Agricultural and Biological Chemistry 55, 1907-1911.

YAO, R. C. \& HsIEH, D. P. H. (1974). Step of dichlorvos inhibition in the pathway of aflatoxin biosynthesis. Applied Microbiology 28, 5257. 